\title{
Stokes Antenna Temperatures
}

David G. Long

david_long@byu.edu

Jeffrey R. Piepmeier

Eni G. Njoku

Follow this and additional works at: https://scholarsarchive.byu.edu/facpub

Part of the Electrical and Computer Engineering Commons

\section{Original Publication Citation}

Piepmeier, J. R., D. G. Long, and E. G. Njoku. "Stokes Antenna Temperatures." Geoscience and Remote Sensing, IEEE Transactions on 46.2 (28): 516-27

\section{BYU ScholarsArchive Citation}

Long, David G.; Piepmeier, Jeffrey R.; and Njoku, Eni G., "Stokes Antenna Temperatures" (2008). Faculty Publications. 208.

https://scholarsarchive.byu.edu/facpub/208

This Peer-Reviewed Article is brought to you for free and open access by BYU ScholarsArchive. It has been accepted for inclusion in Faculty Publications by an authorized administrator of BYU ScholarsArchive. For more information, please contact ellen_amatangelo@byu.edu. 


\title{
Stokes Antenna Temperatures
}

\author{
Jeffrey R. Piepmeier, Member, IEEE, David G. Long, Senior Member, IEEE, and Eni G. Njoku, Fellow, IEEE
}

\begin{abstract}
The growing importance of polarimetric radiometers has led to the need for a detailed theory for Stokes antenna temperatures. In this paper, we provide a full Stokes vector formulation of an antenna temperature that accounts for the entire antenna pattern, which includes polarization mixing in the main-beam and sidelobe effects. To derive the Stokes antenna temperatures, we follow the conventional methods in the Earth remote sensing literature while relying on a coherency algebra approach from radio astronomy. Connections and parallels to the conventional approaches are noted along the way. We also introduce generalizations of beam efficiency and cross polarization for use with polarimetric radiometers. These provide important metrics in the design of future systems.
\end{abstract}

Index Terms-Jones matrix, Mueller matrix, polarimetry, polarization, radiometry, Stokes parameters.

\section{INTRODUCTION}

$\mathbf{P}$ OLARIMETRIC radiometers, or microwave polarimeters, are becoming increasingly important for Earth remote sensing. Whereas conventional dual-polarization radiometers only measure vertical and horizontal polarized brightness temperatures, a polarimetric radiometer also measures the third and fourth Stokes parameters. Polarimetric capabilities have enabled the sensing of ocean-surface wind directions, as well as the enhanced measurements of wind speed, among other applications. Airborne radiometers based on polarization combiners and analog and digital correlators were used to make some of the first nonnadir polarimetric measurements of the sea surface, e.g., [1]-[4]. The first spaceborne polarimeter for Earth observation, called WindSat, was designed for oceansurface wind-vector remote sensing and has produced compelling results [5]. An investigation of antenna pattern effects in polarimetric radiometers was performed for the WindSat development by examining the effects of antenna main-beam cross polarization imperfections on the retrieval of the oceansurface wind direction [6].

To extend the application of polarimetry to other geophysical observations, implementation at L-band is planned for three

Manuscript received November 6, 2006; revised July 16, 2007. This work was supported by the National Aeronautics and Space Administration (NASA) Hydrosphere State (Hydros) Earth System Science Pathfinder Mission. The research described in this paper was carried out in part at the Jet Propulsion Laboratory, California Institute of Technology, under contract with NASA.

J. R. Piepmeier is with the Goddard Space Flight Center, National Aeronautics and Space Administration, Greenbelt, MD 20771-0001 USA (e-mail: jeff.piepmeier@nasa.gov).

D. G. Long is with the Department of Electrical and Computer Engineering, Brigham Young University, Provo, UT 84602 USA.

E. G. Njoku is with the Jet Propulsion Laboratory, California Institute of Technology, Pasadena, CA 91109 USA.

Digital Object Identifier 10.1109/TGRS.2007.909597 upcoming sea-surface salinity and soil moisture spaceborne missions: 1) Soil Moisture and Ocean Salinity (SMOS) [7]; 2) Aquarius [8]; and 3) Soil Moisture Active/Passive [9] (which was preceded by the Hydrosphere State (Hydros) study [10] ${ }^{1}$ ). For these missions, polarimetric measurements at L-band will primarily be used for correcting polarization rotations, including the ionospheric Faraday rotation, which is particularly important for the precision Aquarius radiometer [11], [12]. Because SMOS is a 2-D spatial interferometer, the instrument polarization is necessarily mismatched with the Earth's natural basis in most directions [13]. Thus, measurement of the Stokes visibilities is necessary for the complete correction of the instrumental polarization basis rotation [14]. Electronic polarization basis rotation can also be used to correct the effects induced by the rotations of an observing platform [15], [16] or of a reflector relative to a fixed feed horn [17]. This latter application was planned for the Hydros radiometer, where a large 6-m aperture precipitated the use of the fixed-feed rotating-reflector configuration [18]. Although polarization rotation correction is the primary motivation for L-band polarimetric measurements from space, recent field experiments suggest the presence of significant anisotropic and polarimetric signatures over land surfaces [19], [20]. Further investigation of spaceborne polarimetric measurements of land, sea, and ice surfaces are sure to provide interesting new data and insights into the characteristics and phenomena of these surfaces (e.g., [21]). Whereas polarimetry for Earth observation has been developed only over the previous two decades, it has been used in radio astronomy since at least the 1950s. The applications range from detecting the temporalpolarization signatures of pulsars to mapping the polarization of the cosmic microwave background [22], [23]. Despite this breadth of potential applications, however, a development of Stokes antenna temperatures is not yet available in the literature.

A standard microwave remote sensing text [24] introduces a scalar formulation for the antenna temperature as

$$
T_{\mathrm{A}}=\frac{A_{e}}{\lambda^{2}} \int_{4 \pi} F_{n} T_{\mathrm{B}} d \Omega
$$

where $T_{\mathrm{B}}$ is the brightness temperature distribution, $F_{n}$ is the normalized antenna pattern, $A_{e}$ is the effective aperture area of the antenna, and $\lambda$ is the wavelength of the mean frequency of interest. Both $T_{\mathrm{B}}$ and $F_{n}$ are functions of direction. It should be noted that the brightness temperature is related to the incident-specific intensity by the Rayleigh-Jeans

\footnotetext{
${ }^{1}$ See http://hydros.gsfc.nasa.gov as of July 16, 2007.
} 
low-frequency approximation to Planck's radiation law [25] by

$$
T_{\mathrm{B}}=\frac{\lambda^{2}}{k_{\mathrm{B}}} I
$$

where $k_{\mathrm{B}}$ is Boltzmann's constant, $\lambda$ is the wavelength, and $I$ is the specific intensity. Specific intensity has SI units of watts per hertz per steradian per square meter $\left(\mathrm{W} \cdot \mathrm{Hz}^{-1} \cdot \mathrm{sr}^{-1} \cdot \mathrm{m}^{-2}\right)$. A polarimetric version of (1) is needed-a theory of Stokes antenna temperatures.

The reception of partially polarized radiation by a singleport antenna was formulated several decades ago by Ko [26]. His theory for an effective aperture and antenna temperature was based on the coherency matrix used throughout the optics literature [27]. In another communication, Ko derived what he coined the Stokes vector of the antenna, which can be used to determine the available power at an antenna port due to an incident field in terms of its Stokes vector [28]. The coherency matrix formulation of an effective aperture is the basis of polarized antenna temperature formulations in both radio astronomy and remote sensing literature. Indeed, in their fundamental work on the recovery of dual-polarized brightness temperatures, Claassen and Fung, in part, used Ko's theory to compute dual-polarized antenna temperatures [29]. They were the first to address the effects of antenna cross polarization and the polarization mismatch that occurs between the antenna pattern and the Earth's surface. The antenna pattern correction (APC) algorithm for the Scanning Multichannel Microwave Radiometer was based on this formulation [30]. With but one exception to date, the APC literature only covers scalar and dual-polarization cases (the exception deals with the full Stokes vector, but only within the main beam and not in the sidelobe and back lobe [6]). However, the vector radiative transfer (VRT) theory, which is used to model brightness temperature distributions, has been rigorously developed to handle the full Stokes vector [25], [31], [32]. The polarimetric connection between the VRT theory and Earth observation instrument models has yet to be fully made and is developed here.

This paper is an update to the important works of the past and contains the development of a full Stokes vector formulation of an antenna temperature. We follow the derivation method of Claassen and Fung [29]; however, in that 1974 work, only the vertical and horizontal polarized brightness temperatures were derived, as the utility of the third and fourth Stokes parameters in passive microwave remote sensing were unknown at that time. Thus, we use their approach to derive similar relations for all of the four Stokes parameters while relying on the coherency algebra approach set forth by Hamaker et al. [33], along with its relationship with Stokes algebra [34]. The parallels and similarities to this past work are drawn along the way to provide a concise and, hopefully, clear derivation and expression of Stokes antenna temperatures. Finally, some new interpretations of antenna pattern properties such as beam efficiency and cross polarization for polarimetric radiometers are discussed and applied to polarization-correlating and polarization-adding radiometers.

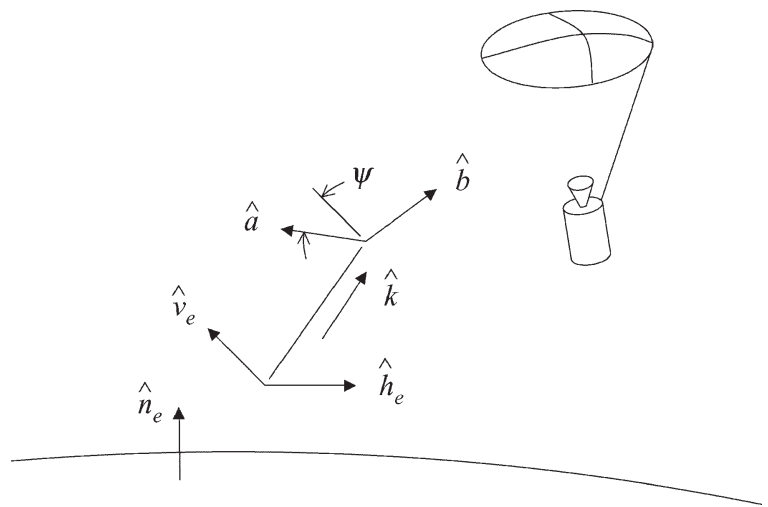

Fig. 1. Earth $\left(\widehat{v}_{e}-\widehat{h}_{e}\right)$ and antenna $(\widehat{a}-\widehat{b})$ polarization bases for a wave propagating along the $\widehat{k}$-direction.

\section{INCIDENT FIELDS}

Quasi-monochromatic plane waves that arise from a brightness distribution propagate toward the antenna in direction $\widehat{k}$. The electric field at position $\vec{r}$ is

$$
\bar{e}(t, \vec{r}, \widehat{k})=\left[\begin{array}{l}
e_{v}(t, \widehat{k}) \\
e_{h}(t, \widehat{k})
\end{array}\right] e^{-j \frac{2 \pi}{\lambda} \widehat{k} \cdot \vec{r}}
$$

where the vector is in the Earth's natural polarization basis $\widehat{v}_{e}-\widehat{h}_{e}$. The quantity $\bar{e}$ is a time-varying vector phasor expressed in a root-mean-square amplitude. We define the polarization vectors such that the horizontal vector is perpendicular to the Earth normal as

$$
\begin{aligned}
\widehat{h}_{e} & =\frac{\widehat{k} \times \widehat{n}_{e}}{\left|\widehat{k} \times \widehat{n}_{e}\right|} \\
\widehat{v}_{e} & =\widehat{h}_{e} \times \widehat{k}
\end{aligned}
$$

where $\widehat{n}_{e}$ is the local Earth's surface normal unit vector (see Fig. 1).

The intensity and polarization state of this wave can be described by its coherency vector [33]

$$
\bar{E}(\widehat{k})=\left\langle\bar{e} \otimes \bar{e}^{*}\right\rangle=\left[\begin{array}{c}
\left\langle\left|e_{v}\right|^{2}\right\rangle \\
\left\langle e_{v} e_{h}^{*}\right\rangle \\
\left\langle e_{h} e_{v}^{*}\right\rangle \\
\left\langle\left|e_{h}\right|^{2}\right\rangle
\end{array}\right]
$$

where the operator $\otimes$ indicates an outer product, and the angle brackets $\langle\cdot\rangle$ denote an ensemble average of the argument. Because the electric field amplitude random processes are stationary and ergodic, there is no need to carry the time argument $t$. The direction argument has been dropped inside the coherency vector for convenience.

At this point, a few comments about the notation are appropriate. Three-space vectors such as $\widehat{k}$ are written using lowercase letters with either an arrow or a caret, the latter indicating unity length. We use lowercase letters with a single overbar, e.g., $\bar{e}$, to denote two-by-one vectors such as the electric field. Two-by-two matrices are likewise indicated using lowercase letters with a double overbar. Four-by-one vectors, such as the coherency vector $\bar{E}$, are written using uppercase letters and a 
single overbar, whereas four-by-four matrices are written using uppercase letters and a double overbar. Other variables, either upper- or lowercase with no decoration, are scalars.

Although the coherency vector provides a convenient form and an associated algebra, as found hereafter, the modified Stokes vector is commonly used in microwave remote sensing. For this quasi-monochromatic wave of bandwidth $d f$ traveling through differential solid angle $d \Omega$, it is [25]

$$
\bar{I} d \Omega d f=\left[\begin{array}{c}
I_{v} \\
I_{h} \\
U \\
V
\end{array}\right] d \Omega d f=\frac{1}{\eta_{0}}\left[\begin{array}{c}
\left\langle\left|e_{v}\right|^{2}\right\rangle \\
\left\langle\left|e_{h}\right|^{2}\right\rangle \\
2 \operatorname{Re}\left\langle e_{v} e_{h}^{*}\right\rangle \\
2 \operatorname{Im}\left\langle e_{v} e_{h}^{*}\right\rangle
\end{array}\right]
$$

where the modified Stokes parameters are quantities of the specific intensity. The modified Stokes vector can also be found from the coherency vector by a transformation matrix $\overline{\bar{T}}_{\mathrm{MC}}$ as

$$
\bar{I} d \Omega d f=\frac{1}{\eta_{0}} \overline{\bar{T}}_{\mathrm{MC}} \bar{E}
$$

where

$$
\overline{\bar{T}}_{\mathrm{MC}}=\left[\begin{array}{cccc}
1 & 0 & 0 & 0 \\
0 & 0 & 0 & 1 \\
0 & 1 & 1 & 0 \\
0 & -j & j & 0
\end{array}\right] .
$$

The subscript MC indicates that the transformation is from coherency vector coordinates to modified Stokes vector coordinates. Sometimes, it is useful to use the true Stokes vector $[I, Q, U, V]^{T}$, where $I=I_{v}+I_{h}$, and $Q=I_{v}-I_{h}$. Transformation matrices that deal with the true Stokes vector are given in the Appendix.

The modified Stokes vector of brightness temperatures (in kelvins) is defined by applying the Rayleigh-Jeans law (2) to the modified Stokes vector of the specific intensity as

$$
\bar{T}_{\mathrm{B}}=\frac{\lambda^{2}}{k_{\mathrm{B}}} \bar{I} .
$$

The symbols used for individual modified Stokes brightness temperatures with the vector $\bar{T}_{\mathrm{B}}$ vary within the literature, with a summary given in the Appendix. In this paper, the following notation is used:

$$
\bar{T}_{\mathrm{B}}=\left[\begin{array}{c}
T_{\mathrm{B}, v} \\
T_{\mathrm{B}, h} \\
T_{\mathrm{B}, U} \\
T_{\mathrm{B}, V}
\end{array}\right]
$$

\section{RECEIVED POWER}

The receiving antenna is defined to be dually polarized to receive power in an arbitrary orthogonal polarization basis $\widehat{a}-\widehat{b}$, which forms a right-handed system with $\widehat{k}$, such that $\widehat{k}=\widehat{a} \times \widehat{b}$ (see Fig. 1). The use of Ludwig's third definition of polarization basis [35] for the antenna is standard. The choice of antenna polarization basis is important because it defines the reference frame of the Stokes parameters. In practice, it is logical to coalign $\widehat{a}-\widehat{b}$ with $\widehat{v}_{e}-\widehat{h}_{e}$ along the antenna boresight, but this is not required. Whereas the antenna basis might be aligned to the Earth basis, which is a mere mathematical construct, the antenna feed polarization vectors are not required to be so. Two such important cases used on WindSat [5], namely, $\pm 45^{\circ}$ linear polarization and dual circular polarization, are illustrated in Section V. The development in this section makes no assumption as to the particular feed polarization in use.

The open-circuit voltage $v_{\mathrm{Oc}}$ at a single antenna port due to an incident wave is

$$
v_{\mathrm{oc}}=\bar{l}_{e} \cdot \bar{e}
$$

where $\bar{l}_{e}$ is the complex vector effective length of the antenna [36]. The effective length is an important concept for describing the reception and transmission of partially polarized radiation by an antenna [26]. Indeed, it is the basis of the past works upon which this paper builds [6], [29], [30]. The open-circuit voltages at the ports of a dual-polarization (and lossless) antenna due to an incident wave from the $\widehat{k}$-direction are found using the following Jones matrix notation [27]:

$$
\bar{v}_{\mathrm{oc}}(\widehat{k})=\overline{\bar{l}}(\widehat{k}) \overline{\bar{p}}(\psi) \bar{e}(\widehat{k})
$$

where $\overline{\bar{l}}$ is a Jones matrix [27] of effective lengths defined as

$$
\overline{\bar{l}}(\widehat{k})=\left[\begin{array}{ll}
l_{a a} & l_{a b} \\
l_{b a} & l_{b b}
\end{array}\right] .
$$

A single row of this Jones matrix contains the length vector of a single-port antenna utilized by Ko [26]. If the antenna ports (as determined by feed design) are coaligned with the $\widehat{a}-\widehat{b}$ polarizations, then the aforementioned Jones matrix can be interpreted so that the copolarization length patterns for the $\widehat{a}$ and $\widehat{b}$ ports are $l_{a a}$ and $l_{b b}$, respectively. The two other patterns $l_{a b}$ and $l_{b a}$ are the cross polarization patterns. For such a case, the ideal Jones matrix is the identity matrix. As previously pointed out, the antenna and Earth bases are not required to be aligned within the main beam, and furthermore, as noted by Claassen and Fung [29], polarization misalignment is generally always present outside the main beam. To align the Earth basis $\widehat{v}_{e}-\widehat{h}_{e}$ with the antenna basis $\widehat{a}-\widehat{b}$, the matrix $\overline{\bar{p}}$ rotates the polarization through the angle $\psi$ (as illustrated in Fig. 1). Thus, we have

$$
\overline{\bar{p}}(\psi)=\left[\begin{array}{ll}
\widehat{v}_{e} \cdot \widehat{a} & \widehat{h}_{e} \cdot \widehat{a} \\
\widehat{v}_{e} \cdot \widehat{b} & \widehat{h}_{e} \cdot \widehat{b}
\end{array}\right]=\left[\begin{array}{cc}
\cos \psi & \sin \psi \\
-\sin \psi & \cos \psi
\end{array}\right] .
$$

The differential power that is available from the open-circuit voltage at a single port (11) is

$$
d W=\frac{1}{4 Z_{0}}\left\langle v_{\mathrm{oc}} v_{\mathrm{oc}}^{*}\right\rangle
$$

where $Z_{0}$ is the characteristic impedance of the antenna port. Likewise, the differential available power coherency vector is defined as

$$
d \bar{W}(\widehat{k})=\frac{1}{4 Z_{0}}\left\langle\bar{v}_{\mathrm{oc}} \otimes \bar{v}_{\mathrm{oc}}^{*}\right\rangle .
$$


Substituting (12) into (16) yields

$$
d \bar{W}=\frac{1}{4 Z_{0}} \overline{\bar{L}} \overline{\bar{P}} \bar{E}
$$

where

$$
\begin{aligned}
& \overline{\bar{L}}=\left(\overline{\bar{l}} \otimes \overline{\bar{l}}^{*}\right) \\
& \overline{\bar{P}}=\left(\overline{\bar{p}} \otimes \overline{\bar{p}}^{*}\right) .
\end{aligned}
$$

Thus, the coherency vector of the antenna output is a simple transformation of that of the incident wave. Because the thermal radiation from the Earth is spatially incoherent in the far zone of the antenna, the total available power can be found by integrating over all directions, i.e.,

$$
\bar{W}=\int_{4 \pi} d \bar{W}
$$

or, in terms of the incident brightness distribution, by substituting (7) into (17)

$$
\bar{W}=\int_{4 \pi} \frac{\eta_{0}}{4 Z_{0}} \overline{\bar{L}} \overline{\bar{P}} \overline{\bar{T}}_{\mathrm{MC}}^{-1} \bar{I} d \Omega d f .
$$

Before continuing further to examine the transformations $\overline{\bar{L}}$ and $\overline{\bar{P}}$, the modified Stokes vector of antenna noise temperatures is introduced.

\section{Antenna Temperatures}

For a lossless dual-polarization antenna, the Stokes vector of antenna temperatures $\bar{T}_{\mathrm{A}}$ is related to the available power by the following Nyquist relationship:

$$
k_{\mathrm{B}} \bar{T}_{\mathrm{A}} d f=\overline{\bar{T}}_{\mathrm{MC}} \bar{W} .
$$

(For the lossy case, the apparent aperture temperature is used rather than strictly an antenna temperature.) By substituting (19) into (20) and applying (9), we can write the modified Stokes vector of the antenna temperatures in terms of an integral over a weighted distribution of brightness temperatures as

$$
\bar{T}_{\mathrm{A}}=\frac{\eta_{0}}{4 Z_{0} \lambda^{2}} \int_{4 \pi} \overline{\bar{T}}_{\mathrm{MC}} \overline{\bar{L}} \overline{\overline{P T}}_{\mathrm{MC}}^{-1} \bar{T}_{\mathrm{B}} d \Omega
$$

where $\lambda=c / f$. The matrix portion of the integrand in (21) can be decomposed into two operators as follows:

$$
\overline{\bar{T}}_{\mathrm{MC}} \overline{\bar{L}} \overline{\overline{P T}}_{\mathrm{MC}}^{-1}=\left[\overline{\bar{T}}_{\mathrm{MC}} \overline{\bar{L}} \overline{\bar{T}}_{\mathrm{MC}}^{-1}\right]\left[\overline{\bar{T}}_{\mathrm{MC}} \overline{\overline{P T}}_{\mathrm{MC}}^{-1}\right] .
$$

The premultiplications and postmultiplications by $\overline{\bar{T}}_{\mathrm{MC}}$ and $\overline{\bar{T}}_{\text {MC }}^{-1}$ convert a $4 \times 4$ coherency vector operator to a modified Stokes vector operator, or a Mueller matrix. A Mueller matrix transforms an input Stokes vector to an output Stokes vector and is used, i.e., in the VRT theory [25], to describe electromagnetic scattering. They have also been used to model the radio telescope at the Aercibo Observatory [37] and are used in Section V to apply the results of this paper to the different feed polarizations.

The first operator in (22) contains the directional receiving properties of the antenna, and the second has a polarization basis rotation. It can be shown that the latter $\overline{\bar{T}}_{\mathrm{MC}} \overline{\bar{P}}(\psi) \overline{\bar{T}}_{\mathrm{MC}}^{-1}$ is exactly the polarization basis rotation operator $\overline{\bar{R}}(\psi)$ for the modified Stokes vector defined in [25] as

$$
\overline{\bar{R}}(\psi)=\left[\begin{array}{cccc}
\cos ^{2} \psi & \sin ^{2} \psi & \frac{1}{2} \sin 2 \psi & 0 \\
\sin ^{2} \psi & \cos ^{2} \psi & -\frac{1}{2} \sin 2 \psi & 0 \\
-\sin 2 \psi & \sin 2 \psi & \cos 2 \psi & 0 \\
0 & 0 & 0 & 1
\end{array}\right] .
$$

The second operator $\overline{\bar{T}}_{\mathrm{MC}} \overline{\bar{L}} \overline{\bar{T}}_{\mathrm{MC}}^{-1}$ contains the matrix $\overline{\bar{L}}$, which is the outer product composition of the lengths of matrix $\bar{l}$. The effective lengths can be defined in terms of the normalized voltage antenna patterns $\overline{\bar{f}}_{n}$ as

$$
\overline{\bar{l}}(\widehat{k})=2\left(\frac{Z_{0}}{\eta_{0}}\right)^{\frac{1}{2}} \overline{\bar{a}}_{e} \overline{\bar{f}}_{n}(\widehat{k})
$$

where

$$
\overline{\bar{f}}_{n}(\widehat{k})=\left[\begin{array}{ll}
f_{n, a a} & f_{n, a b} \\
f_{n, b a} & f_{n, b b}
\end{array}\right]
$$

and $\overline{\bar{a}}_{e}$ is a diagonal matrix

$$
\overline{\bar{a}}_{e}=\left[\begin{array}{cc}
A_{e, a}^{1 / 2} & 0 \\
0 & A_{e, b}^{1 / 2}
\end{array}\right]
$$

of effective aperture areas $A_{e, x}$ for antenna polarization $x=$ $a$ or $b$. The matrix $\overline{\bar{f}}$ describes the receiving properties of an equivalent antenna with a unit effective length. The elements $f_{n, a b}(x y=a a, a b, b a$, or $b b)$ are unitless and normalized such that $\max \left(\left|f_{n, a a}\right|^{2}+\left|f_{n, a b}\right|^{2}\right)=\max \left(\left|f_{n, b b}\right|^{2}+\left|f_{n, b a}\right|^{2}\right) \equiv 1$.

Expanding the outer product with this definition yields

$$
\overline{\bar{L}}=4 \frac{Z_{0}}{\eta_{0}}\left(\overline{\bar{a}}_{e} \otimes \overline{\bar{a}}_{e}\right)\left(\overline{\bar{f}}_{n} \otimes \overline{\bar{f}}_{n}^{*}\right)
$$

where

$$
\begin{aligned}
\overline{\bar{a}}_{e} & \otimes \overline{\bar{a}}_{e} \\
& =\left[\begin{array}{cccc}
A_{e, a} & 0 & 0 & 0 \\
0 & \sqrt{A_{e, a} A_{e, b}} & 0 & 0 \\
0 & 0 & \sqrt{A_{e, a} A_{e, b}} & 0 \\
0 & 0 & 0 & A_{e, b}
\end{array}\right] \\
\overline{\bar{f}}_{n} & \otimes \overline{\bar{f}}_{n}^{*} \\
= & {\left[\begin{array}{cccc}
\left|f_{n, a a}\right|^{2} & f_{n, a a} f_{n, a b}^{*} & f_{n, a b} f_{n, a a}^{*} & \left|f_{n, a b}\right|^{2} \\
f_{n, a a} f_{n, b a}^{*} & f_{n, a a} f_{n, b b}^{*} & f_{n, a b} f_{n, b a}^{*} & f_{n, a b} f_{n, b b}^{*} \\
f_{n, b a} f_{n, a a}^{*} & f_{n, b a} f_{n, b a}^{*} & f_{n, b b} f_{n, a a}^{*} & f_{n, b b} f_{n, a b}^{*} \\
\left|f_{n, b a}\right|^{2} & f_{n, b a} f_{n, b b}^{*} & f_{n, b b} f_{n, b a}^{*} & \left|f_{n, b b}\right|^{2}
\end{array}\right] . }
\end{aligned}
$$


Note that the top and bottom rows of $\overline{\bar{L}}$, excluding the impedance scaling factor, are the vector expressions of Ko's definition of effective aperture using the coherency matrix [28]. The middle two rows deal with the cross correlations between the two antenna polarizations, which are necessarily measured to find the third and fourth Stokes parameters. The equivalent Mueller matrix of $\overline{\bar{L}}$ is

$$
\overline{\bar{T}}_{\mathrm{MC}} \overline{\bar{L}} \overline{\bar{T}}_{\mathrm{MC}}^{-1}=4 \frac{Z_{0}}{\eta_{0}} \overline{\bar{A}}_{e} \overline{\bar{F}}_{n}
$$

where the antenna effective area matrix is

$$
\overline{\bar{A}}_{e}=\left[\begin{array}{cccc}
A_{e, a} & 0 & 0 & 0 \\
0 & A_{e, b} & 0 & 0 \\
0 & 0 & \sqrt{A_{e, a} A_{e, b}} & 0 \\
0 & 0 & 0 & \sqrt{A_{e, a} A_{e, b}}
\end{array}\right]
$$

and the normalized antenna radiation pattern matrix is expressed in (32), shown at the bottom of the page.

Note that the upper left-hand $2 \times 2$ submatrix contains the normalized radiation patterns that are conventionally associated with dual-polarized antennas. The first and second rows of the matrix product $\overline{\bar{A}}_{e} \overline{\bar{F}}_{n}$ are equivalent to what Ko defines as the Stokes vector for the antenna [28]. In addition, consider the third and fourth elements of the first or second rows of $\overline{\bar{F}}_{n}$. The ratio of these two elements gives the cross polarization phase singled out by Claassen and Fung [29] as

$$
\tan \beta=\frac{\operatorname{Im} f_{n, a a} f_{n, a b}^{*}}{\operatorname{Re} f_{n, a a} f_{n, a b}^{*}} .
$$

Furthermore, the matrix $\overline{\bar{F}}_{n}$ is identical in form to a phase matrix found in the VRT theory [38]. Composed of bistatic scattering amplitude functions, the phase matrix describes how incident modified Stokes vectors are scattered by a distribution of particles. In this sense, the antenna can be thought of as scattering incident brightness temperature vectors $\bar{T}_{\mathrm{B}}(\widehat{k})$ into the antenna temperature vector $\bar{T}_{\mathrm{A}}$, as governed by the normalized phase matrix in (32).

Finally, substituting (23) and (30) into (21) yields

$$
\bar{T}_{\mathrm{A}}=\frac{1}{\lambda^{2}} \overline{\bar{A}}_{e} \int_{4 \pi} \overline{\bar{F}}_{n}(\widehat{k}) \overline{\bar{R}}(\psi) \bar{T}_{\mathrm{B}}(\widehat{k}) d \Omega .
$$

First, compare this equation for antenna temperature with (1), which is the scalar version found in [24]. Equation (34) contains the essential elements of effective aperture, normalized antenna pattern, and incident brightness temperature distribution, albeit in the modified Stokes vector and Mueller matrix form. Second, compare this with the single-port antenna version of Claassen and Fung [29]. It contains the key elements of cross polarization amplitude and phase, as well as the polarization mismatch between the antenna pattern and the Earth's surface. In fact, the first and second rows comprise their antenna temperature model. The expression here, however, goes a step further to include the reception of the full modified Stokes vector. The extension of previous scalar and vector developments by using the outer product to include the cross correlation terms is essential to understanding the reception of the full Stokes vector by a dual-polarization antenna.

Finally, the development, thus far, has not explicitly addressed the frequency response of the antenna. In principle, the antenna temperature model (34) is a function of frequency. If the antenna is significantly wideband with a fluctuating response, or the upwelling brightness temperature varies with the frequency over the operating band, then an integration over the frequency is needed to compute the aggregate response. In general, the integration should be over $\bar{T}_{\mathrm{A}}(f)$. If, however, $\bar{T}_{\mathrm{B}}$ is constant over the band of interest, the integration can be done over the antenna pattern matrix $\lambda^{-2} \overline{\bar{A}}_{e} \overline{\bar{F}}_{n}$, where $\lambda=c / f$, and both $\overline{\bar{A}}_{e}$ and $\overline{\bar{F}}_{n}$ can vary with frequency.

\section{ApPlication}

\section{A. Polarimetric Radiometers}

Microwave thermal emission from the Earth's surface lends itself to a natural polarization basis $\widehat{v}_{e}-\widehat{h}_{e}$, as described in Section II. The measurements of surface-sensing radiometers are typically reported in this basis. Thus, when modeling the antenna temperatures, it is meaningful to define the antenna polarization basis $\widehat{a}-\widehat{b}$ such that it coaligns with the natural Earth basis along the center of the main beam (this basis is denoted hereafter by the symbols $\widehat{v}-\widehat{h}$.) A dual-polarization feed aligned with $\widehat{v}-\widehat{h}$ produces an antenna temperature vector aligned to the polarization basis of the Earth's surface along the antenna boresight. Conventional dual-polarization radiometers with conical scanning use this configuration. This arrangement is also typical for direct-correlation polarimetric radiometers that employ cross correlation receivers for measuring the third and fourth Stokes parameters (e.g., [3] and [4]). For example, the third Stokes antenna temperature can be measured by cross correlating the $v$ - and $h$-polarization antenna voltages received over bandwidth $B$ as

$$
T_{\mathrm{A}, U}=\frac{\operatorname{Re}\left\langle v_{\mathrm{oc}, v} v_{\mathrm{oc}, h}^{*}\right\rangle}{2 Z_{0} k_{\mathrm{B}} B} .
$$

The aforementioned equation comes directly from (20). The feed polarization coalignment is also typical for those receivers

$$
\overline{\bar{F}}_{n}=\left[\begin{array}{cccc}
\left|f_{n, a a}\right|^{2} & \left|f_{n, a b}\right|^{2} & \operatorname{Re} f_{n, a a} f_{n, a b}^{*} & -\operatorname{Im} f_{n, a a} f_{n, a b}^{*} \\
\left|f_{n, b a}\right|^{2} & \left|f_{n, b b}\right|^{2} & \operatorname{Re} f_{n, b b} f_{n, b a}^{*} & \operatorname{Im} f_{n, b b} f_{n, b a}^{*} \\
2 \operatorname{Re} f_{n, a a} f_{n, b a}^{*} & 2 \operatorname{Re} f_{n, b b} f_{n, a b}^{*} & \operatorname{Re} f_{n, a a} f_{n, b b}^{*}+\operatorname{Re} f_{n, a b} f_{n, b a}^{*} & -\operatorname{Im} f_{n, a a} f_{n, b b}^{*}+\operatorname{Im} f_{n, a b} f_{n, b a}^{*} \\
2 \operatorname{Im} f_{n, a a} f_{n, b a}^{*} & -2 \operatorname{Im} f_{n, b b} f_{n, a b}^{*} & \operatorname{Im} f_{n, a a} f_{n, b b}^{*}+\operatorname{Im} f_{n, a b} f_{n, b a}^{*} & \operatorname{Re} f_{n, a a} f_{n, b b}^{*}-\operatorname{Re} f_{n, a b} f_{n, b a}^{*}
\end{array}\right]
$$


based on hybrid couplers that are used for synthesizing $\pm 45^{\circ}$ linear or left- and right-hand circular polarizations (LHCP and RHCP) (e.g., [2]). The third Stokes parameter can be measured, using a $180^{\circ}$ hybrid coupler to synthesize $\pm 45^{\circ}$ linear polarizations from $v$ and $h$, by

$$
T_{\mathrm{A}, U}=T_{\mathrm{A},+45^{\circ}}-T_{\mathrm{A},-45^{\circ}}
$$

where

$$
T_{\mathrm{A}, \pm 45^{\circ}}=\frac{\left|v_{\mathrm{oc}, v} \pm v_{\mathrm{oc}, h}\right|^{2}}{8 Z_{0} k_{\mathrm{B}} B} .
$$

A different receiver (and feed) configuration, however, is to use a rotated dual-linear-polarization (or dual-circularpolarization) feed to directly detect the $\pm 45^{\circ}$ linear (or LHCP and RHCP) polarizations. This polarization-adding architecture is implemented on WindSat [5]. To obtain the full Stokes vector, the WindSat instrument uses three feed horns with six totalpower receivers to measure six polarizations: vertical, horizontal, $\pm 45^{\circ}$ linear, LHCP, and RHCP. The third (or fourth) Stokes antenna temperature is determined by differencing the $\pm 45^{\circ}$ linear polarization (or LCHP and RHCP) antenna temperatures. In this case, the antenna feeds transform the antenna polarization basis from $\widehat{v}-\widehat{h}$ to either $\pm 45^{\circ}$ linear polarizations (denoted $\widehat{p}-\widehat{m}$ ) or LHCP and RHCP (denoted $\widehat{l}-\widehat{r}$ ). In the Stokes antenna temperature model presented here, the transforms take the form of a feed Mueller matrix $\overline{\bar{M}}_{F}$, which transforms the antenna temperature reference from the antenna polarization basis to the feed polarization basis [37]

$$
\bar{T}_{F}=\overline{\bar{M}}_{F} \bar{T}_{\mathrm{A}}
$$

Both a set of ideal matrices and a general Mueller matrix for an imperfect feed of arbitrary polarization are presented in [37]. The first WindSat case is a dual linearly polarized feed rotated $45^{\circ}$, which transforms $\widehat{v}-\widehat{h}$ to $\widehat{p}-\widehat{m}$ and has the ideal Mueller matrix

$$
\overline{\bar{M}}_{F}=\left[\begin{array}{cccc}
\frac{1}{2} & \frac{1}{2} & \frac{1}{2} & 0 \\
\frac{1}{2} & \frac{1}{2} & -\frac{1}{2} & 0 \\
-1 & 1 & 0 & 0 \\
0 & 0 & 0 & 1
\end{array}\right]
$$

and a feed-reference antenna temperature vector

$$
\bar{T}_{F}=\left[\begin{array}{c}
T_{F,+45^{\circ}} \\
T_{F,-45^{\circ}} \\
-T_{\mathrm{A}, Q} \\
T_{\mathrm{A}, V}
\end{array}\right]
$$

where $T_{\mathrm{A}, Q}=T_{\mathrm{A}, v}-T_{\mathrm{A}, h}$. It can be shown that the third Stokes antenna temperature is

$$
T_{\mathrm{A}, U}=T_{F,+45}-T_{F,-45} .
$$

The second case is a dual circularly polarized feed, which transforms $\widehat{v}-\widehat{h}$ to $\widehat{l}-\widehat{r}$ and has the ideal Mueller matrix

$$
\overline{\bar{M}}_{F}=\left[\begin{array}{cccc}
\frac{1}{2} & \frac{1}{2} & 0 & \frac{1}{2} \\
\frac{1}{2} & \frac{1}{2} & 0 & -\frac{1}{2} \\
0 & 0 & 1 & 0 \\
-1 & 1 & 0 & 0
\end{array}\right]
$$

and a feed-reference antenna temperature vector

$$
\bar{T}_{F}=\left[\begin{array}{c}
T_{F, \mathrm{LHCP}} \\
T_{F, \mathrm{RHCP}} \\
T_{\mathrm{A}, U} \\
-T_{\mathrm{A}, Q}
\end{array}\right]
$$

It can also be shown that the fourth Stokes antenna temperature is

$$
T_{\mathrm{A}, V}=T_{F, \mathrm{LHCP}}-T_{F, \mathrm{RHCP}} .
$$

An interesting outcome from these results is that the measurements of the third and fourth Stokes antenna temperatures, using either direct-correlating or polarization-adding radiometers, are equal-assuming perfect feeds. That is, antenna pattern effects such as beam efficiency and cross polarization leakage have the same impact on either radiometer architecture. If feed imperfections are considered, this may no longer be the case. Thus, (34) and (38) can be used to model the feedreferenced Stokes antenna temperatures for any antenna and feed polarization basis combination.

\section{B. Antenna Pattern Properties}

In radiometer instrument design, several antenna pattern properties are the key drivers of performance. Beam efficiency is perhaps the most important, followed by antenna cross polarization. In this section, these parameters are derived for a polarimetric radiometer antenna by integrations over the main lobe of the antenna patterns. The Mueller matrix for the antenna patterns can be defined as

$$
\overline{\bar{M}}=\frac{1}{\lambda^{2}} \overline{\bar{A}}_{e} \overline{\bar{F}}_{n}
$$

and the effective aperture areas can be related to pattern solid angles by

$$
\frac{\lambda^{2}}{A_{e, x}}=\Omega_{p, x}
$$

for $x=v$ or $h$, where the pattern solid angles are integrations of the normalized antenna patterns over all $4 \pi$ sr [24], i.e.,

$$
\begin{aligned}
& \Omega_{p, v}=\int_{4 \pi}\left|f_{n, v v}\right|^{2}+\left|f_{n, v h}\right|^{2} d \Omega \\
& \Omega_{p, h}=\int_{4 \pi}\left|f_{n, h h}\right|^{2}+\left|f_{n, h v}\right|^{2} d \Omega .
\end{aligned}
$$

The beam efficiency and cross polarization are found by integrating the appropriate patterns over the main lobe. The 
integrated main-beam matrix is defined as

$$
\overline{\bar{\eta}}_{M}=\iint_{\text {main lobe }} \overline{\bar{M}} d \Omega
$$

with the following notation of individual elements:

$$
\overline{\bar{\eta}}_{M}=\left[\begin{array}{cccc}
\eta_{M, v} & \eta_{M, v h} & \eta_{M, v U} & \eta_{M, v V} \\
\eta_{M, h v} & \eta_{M, h} & \eta_{M, h U} & \eta_{M, h V} \\
\eta_{M, U v} & \eta_{M, U h} & \eta_{M, U} & \eta_{M, U V} \\
\eta_{M, V v} & \eta_{M, V h} & \eta_{M, V U} & \eta_{M, V}
\end{array}\right] .
$$

The first two diagonal elements of this matrix are exactly the definition of beam efficiency [24] defined as

$$
\begin{aligned}
\eta_{M, v} & =\frac{\iint_{\text {main lobe }}\left|f_{n, v v}\right|^{2} d \Omega}{\int_{4 \pi}\left|f_{n, v v}\right|^{2}+\left|f_{n, v h}\right|^{2} d \Omega} \\
\eta_{M, h} & =\frac{\iint\left|f_{n, h h}\right|^{2} d \Omega}{\int_{4 \pi}\left|f_{n, h h}\right|^{2}+\left|f_{n, h v}\right|^{2} d \Omega} .
\end{aligned}
$$

The third and fourth diagonal elements lead us to a new definition of beam efficiency of an antenna for the third and fourth Stokes parameters defined as

$$
\begin{aligned}
\eta_{M, U}= & \iint_{\text {main lobe }} \operatorname{Re} f_{n, v v} f_{n, h h}^{*}+\operatorname{Re} f_{n, v h} f_{n, h v}^{*} d \Omega \\
& \times\left(\int_{4 \pi}\left|f_{n, v v}\right|^{2}+\left|f_{n, v h}\right|^{2} d \Omega\right)^{-1 / 2} \\
& \times\left(\int_{4 \pi}\left|f_{n, h h}\right|^{2}+\left|f_{n, h v}\right|^{2} d \Omega\right)^{-1 / 2} \\
\eta_{M, V}= & \iint_{\text {main lobe }} \operatorname{Re} f_{n, v v} f_{n, h h}^{*}-\operatorname{Re} f_{n, v h} f_{n, h v}^{*} d \Omega \\
& \times\left(\int_{4 \pi}\left|f_{n, v v}\right|^{2}+\left|f_{n, v h}\right|^{2} d \Omega\right)^{-1 / 2} \\
& \times\left(\int_{4 \pi}\left|f_{n, h h}\right|^{2}+\left|f_{n, h v}\right|^{2} d \Omega\right)^{-1 / 2} .
\end{aligned}
$$

The denominator in each of the aforementioned equations is the geometric mean of the pattern solid angles (47). Note that the numerator in the third Stokes parameter beam efficiency is maximized when the $v$ - and $h$-port patterns are in phase with one another. Cross polarization in the antenna patterns does not degrade the beam efficiency as long as it is in phase between the two polarizations. If it is out of phase, cross polarization degrades the third Stokes parameter beam efficiency. Conversely, in-phase (out-of-phase) cross polarization reduces (increases) the fourth Stokes parameter beam efficiency.
A special case is to consider equal antenna patterns for the two polarizations, i.e.,

$$
\begin{aligned}
f_{n, v v} & =f_{n, h h} \\
f_{n, v h} & =f_{n, h v} .
\end{aligned}
$$

The polarimetric beam efficiencies reduce to

$$
\begin{array}{r}
\eta_{M, U}=\frac{\iint_{\text {main lobe }}\left|f_{n, v v}\right|^{2}+\left|f_{n, v h}\right|^{2} d \Omega}{\int_{4 \pi}\left|f_{n, v v}\right|^{2}+\left|f_{n, v h}\right|^{2} d \Omega} \\
\eta_{M, V}=\frac{\iint_{\text {main lobe }}\left|f_{n, v v}\right|^{2}-\left|f_{n, v h}\right|^{2} d \Omega}{\int_{4 \pi}\left|f_{n, v v}\right|^{2}+\left|f_{n, v h}\right|^{2} d \Omega} .
\end{array}
$$

Interestingly, the third Stokes parameter beam efficiency is actually greater than $\eta_{M, v}$ or $\eta_{M, h}$ (unless there is no crosspolarized pattern, in which case the beam efficiencies are all equal). The vertical and horizontal beam efficiencies, by definition, do not include the cross polarization pattern solid angle. Because the third Stokes parameter, however, is detected by an in-phase cross correlation of the signals of the two antenna ports, the cross-polarized portions coherently interfere to increase the detectable cross-power. Thus, the third Stokes parameter beam efficiency includes this cross-polarized pattern solid angle. On the other hand, the fourth Stokes parameter, which is the difference between left- and right-hand circularly polarized intensities, is detected with a quadrature-phase cross correlation. In this case, the cross polarization leakage causes a reduction in detectable cross-power and reduces the beam efficiency. The opposite is true if $f_{n, v h}=-f_{n, h v}$.

Another important antenna property is the integrated mainbeam cross polarization. For the two polarizations, these are

$$
\begin{aligned}
\chi_{v h} & =\frac{\eta_{M, v h}}{\eta_{M, v}} \\
\chi_{h v} & =\frac{\eta_{M, h v}}{\eta_{M, h}}
\end{aligned}
$$

or, when expanded out in integral form, the definitions for the main-beam cross polarization are

$$
\begin{aligned}
& \chi_{v h}=\frac{\iint_{\text {main lobe }}\left|f_{n, v h}\right|^{2} d \Omega}{\iint\left|f_{n, v v}\right|^{2} d \Omega} \\
& \chi_{h v}=\frac{\iint_{\text {main lobe }}\left|f_{n, h v}\right|^{2} d \Omega}{\iint\left|f_{n, h h}\right|^{2} d \Omega} .
\end{aligned}
$$

The analogous cross polarization terms for the third and fourth parameters are

$$
\begin{aligned}
& \chi_{U V}=\frac{\eta_{M, U V}}{\eta_{M, U}} \\
& \chi_{V U}=\frac{\eta_{M, V U}}{\eta_{M, V}}
\end{aligned}
$$


and (likewise expanded in integral form)

$$
\begin{array}{r}
\chi_{U V}=\frac{\iint_{\text {main lobe }}-\operatorname{Im} f_{n, v v} f_{n, h h}^{*}+\operatorname{Im} f_{n, v h} f_{n, h v}^{*} d \Omega}{\iint_{\text {main lobe }} \operatorname{Re} f_{n, v v} f_{n, h h}^{*}+\operatorname{Re} f_{n, v h} f_{n, h v}^{*} d \Omega} \\
\chi_{V U}=\frac{\int_{\text {main lobe }} \operatorname{Im} f_{n, v v} f_{n, h h}^{*}+\operatorname{Im} f_{n, v h} f_{n, h v}^{*} d \Omega}{\iint \operatorname{Re} f_{n, v v} f_{n, h h}^{*}-\operatorname{Re} f_{n, v h} f_{n, h v}^{*} d \Omega} .
\end{array}
$$

Because $U$ and $V$ are not actual polarizations, the phrase "cross polarization" is perhaps a misnomer. The existence of a quadrature-phase relationship between the $v$ - and $h$-polarized patterns causes undesirable mixing of $T_{\mathrm{B}, U}$ and $T_{\mathrm{B}, V}$ into $T_{\mathrm{A}, V}$ and $T_{\mathrm{A}, U}$, respectively. If the patterns are equal, as in (52), then there is no imaginary component to their products, and $\chi_{U V}$ and $\chi_{V U}$ are identically zero. However, even if there were no pattern cross polarizations, a phase imbalance, which is caused, for example, by unequal feed-line lengths, causes mixing of the third and fourth Stokes parameters. In this sense, these cross polarization ratios of the third to the fourth Stokes parameter are also a measure of pattern phase imbalance.

There are eight other off-diagonal elements in (49) that quantify the mixing of the third and fourth parameters into the first two, and conversely, the first and second parameters into the third and fourth. Rather than forming cross polarization ratios, they can be called Stokes parameter mixing ratios. Because geophysical $T_{\mathrm{B}, U}$ and $T_{\mathrm{B}, V}$ are typically much smaller than either $T_{\mathrm{B}, v}$ and $T_{\mathrm{B}, h}$, the mixing of the latter two into the former is examined. The following ratios are defined:

$$
\begin{aligned}
& \chi_{U v}=\frac{\eta_{M, U v}}{\eta_{M, U}} \\
& \chi_{U h}=\frac{\eta_{M, U h}}{\eta_{M, U}} \\
& \chi_{V v}=\frac{\eta_{M, V v}}{\eta_{M, V}} \\
& \chi_{V h}=\frac{\eta_{M, V h}}{\eta_{M, V}} .
\end{aligned}
$$

For the case of equal antenna patterns (52), these ratios are equal in sign and magnitude for $U$ and opposite in sign for $V$

$$
\begin{gathered}
\chi_{U v}=\chi_{U h}=\frac{\iint 2 \operatorname{Re} f_{n, v v} f_{n, v h}^{*} d \Omega}{\iint\left|f_{n, v v}\right|^{2}+\left|f_{n, v h}\right|^{2} d \Omega} \\
\chi_{V v}=-\chi_{V h}=\frac{\iint_{\text {main lobe }} 2 \operatorname{Im} f_{n, v v} f_{n, v h}^{*} d \Omega}{\iint_{\text {main lobe }}\left|f_{n, v v}\right|^{2}-\left|f_{n, v h}\right|^{2} d \Omega} .
\end{gathered}
$$

If there is in-phase cross polarization within an antenna pattern, then there is mixing of $T_{\mathrm{B}, v}$ and $T_{\mathrm{B}, h}$ into $T_{\mathrm{A}, U}$. Likewise, quadrature-phase cross polarization causes mixing into $T_{\mathrm{A}, V}$. A key difference between the two, however, is the sign change in $\chi_{V h}$. Because the sign is the same for the mixing into $T_{\mathrm{A}, U}$, it is the sum of $T_{\mathrm{B}, v}$ and $T_{\mathrm{B}, h}$ that is mixed into $T_{\mathrm{A}, U}$. The sign change causes the difference between $T_{\mathrm{B}, v}$ and $T_{\mathrm{B}, h}$ to be mixed into $T_{\mathrm{A}, V}$.

These sums and differences of the first and second modified Stokes brightness temperatures are actually the first and second (true) Stokes brightness temperatures

$$
\begin{aligned}
T_{\mathrm{B}, I} & =T_{\mathrm{B}, v}+T_{\mathrm{B}, h} \\
T_{\mathrm{B}, Q} & =T_{\mathrm{B}, v}-T_{\mathrm{B}, h}
\end{aligned}
$$

where the subscripts $I$ and $Q$ are the symbols for the first and second Stokes parameters (in units of specific intensity) [22]. Thus, it is the total intensity $T_{\mathrm{B}, I}$ that mixes into $T_{\mathrm{A}, U}$ and the polarization contrast $T_{\mathrm{B}, Q}$ that mixes into $T_{\mathrm{A}, V}$, as described by the following mixing ratios:

$$
\begin{aligned}
\chi_{U I} & =\frac{1}{2}\left(\chi_{U v}+\chi_{U h}\right) \\
\chi_{V Q} & =\frac{1}{2}\left(\chi_{V v}-\chi_{V h}\right) \\
\chi_{U Q} & =\chi_{V I}=0 .
\end{aligned}
$$

See the Appendix for formal details of the transformation of Mueller matrices between modified and true Stokes vector domains.

\section{Example}

During the formulation period of the Hydros mission, patterns of a notional 6-m conical-scanning mesh reflector were modeled to aid in the investigation of radiometer performance and the derivation of antenna requirements. The antenna is an offset-fed paraboloid with a single feed at the focal point and a support boom, extending from near the feed to the center of the reflector. The support boom obstructs the feed's field of view, creating an interesting sidelobe structure and a plane of symmetry about $\phi=0$. The antenna Mueller matrix was computed using simulated antenna patterns and is shown in Fig. 2. The patterns within the matrix are displayed as $k$-space images over $\pm 12.5^{\circ}$ of elevation angle and arranged in the same fashion as the elements of a Muller matrix. Thus, copolarization patterns lay on the diagonal and cross polarization, or Stokes mixing patterns, on the off diagonal. The main-beam region ( 2.5 times the $3-\mathrm{dB}$ beamwidth of $2.5^{\circ}$ ) is indicated by a circle with a $6.25^{\circ}$ diameter seen on the lower left-hand plot. The $2 \times 2$ submatrix in the upper left-hand corner contains the copolarization and cross polarization patterns for the $v$ - and $h$-polarizations that are nonnegative. The remaining patterns, however, can take on both positive and negative values, indicated by the blue and green color scales, respectively. The latter property arises from the cross-correlation operation required to produce the third and fourth Stokes parameters, which often produces negative sidelobes. This phenomenon is also common in imaging interferometer arrays. A fortunate outcome is the summing to zero of positive and negative lobes within the mainbeam region of several Stokes parameter mixing patterns, which is made possible because of the feed's location. Not all mixing parameters sum to zero, i.e., the bottom row of the figure, most 


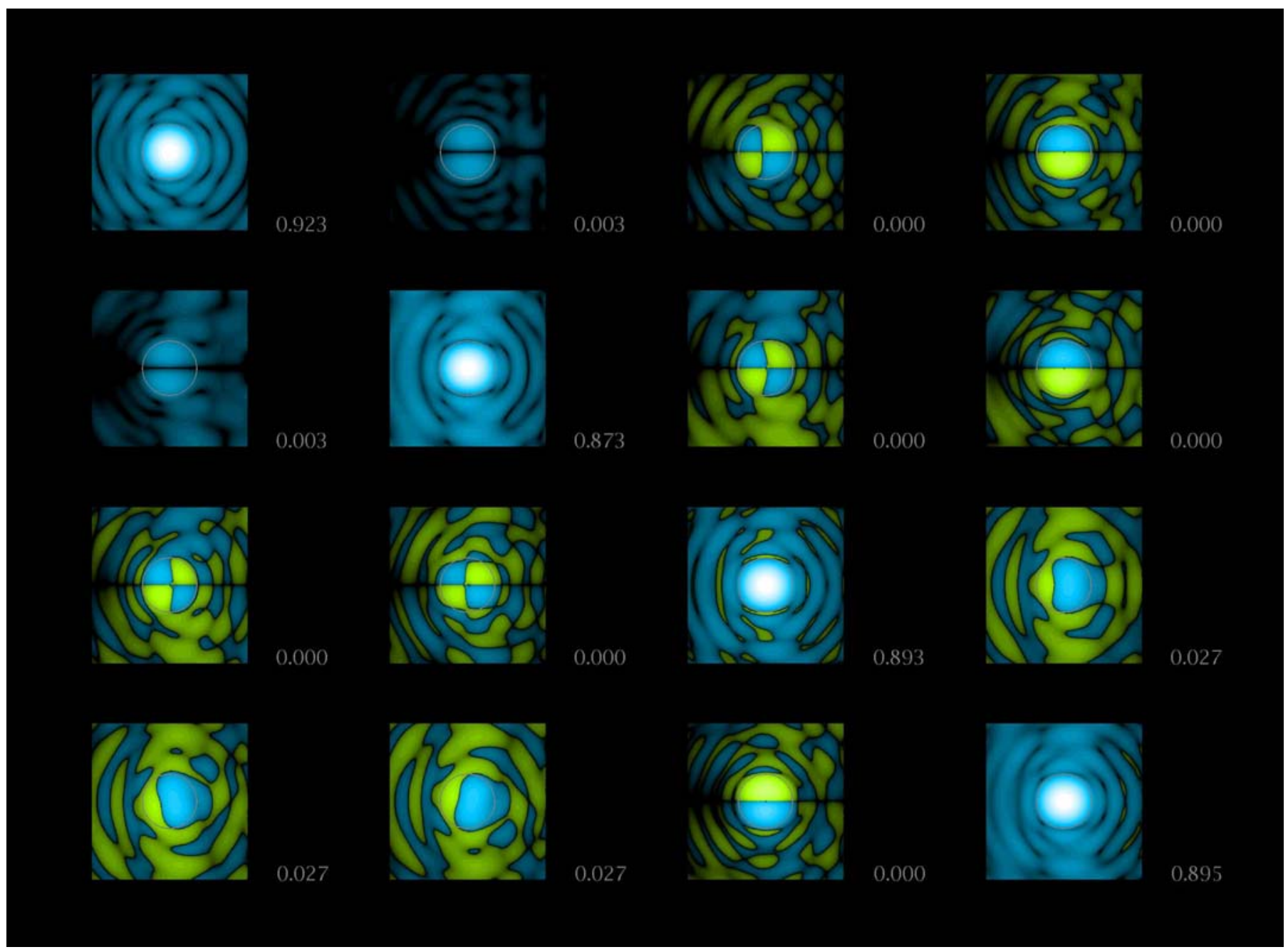

Fig. 2. Graphical Mueller matrix. Intensity indicates relative gain in the log scale. From white (maximum) to black, there is $60 \mathrm{~dB}$ of dynamic range. Blue hue: Positive values. Green hue: Negative values. Numbers: Results of integration over the main-beam region, as indicated by the light-gray circles.

likely because the obstruction of the supporting boom perturbs the reception of circularly polarized signals.

\section{Discussion}

The recently increased usage of polarimetric radiometers in Earth remote sensing revealed the absence of a detailed derivation of Stokes antenna temperatures. In this paper, the expression for the modified Stokes vector of the antenna temperatures has been derived by building on the previous developments. The Stokes antenna temperature integral is remarkably similar to the scalar version found in [24]. Indeed, it contains the essential elements of effective aperture, normalized antenna pattern, and incident brightness temperature distribution, albeit in the modified Stokes vector and Mueller matrix form. To arrive at this Stokes vector form, the coherency algebra approach found in radio astronomy has been utilized [33] to extend the single-port antenna formulation of Claassen and Fung [29]. Our equation contains their key contributions of the effects of cross polarization amplitude and phase, as well as the polarization mismatch between the antenna pattern and the Earth's surface. In fact, the first and second rows of the Stokes antenna temper- ature equation comprise their antenna temperature model for the vertical and horizontal polarizations. The third and fourth rows of our expression contain the key innovation of antenna temperatures for the third and fourth Stokes parameters.

A fortunate by-product of the development was the antenna pattern Mueller matrix, which contained the important polarization properties utilized in [29] in a form that is identical to the Mueller matrix, describing the scattering of Stokes vectors by the distribution of particles in VRT. Further study of this matrix led to the new interpretations of beam efficiency and cross polarization for polarimetric radiometers. Specifically, we define the third and fourth Stokes parameter beam efficiencies for a polarimetric radiometer antenna. We found that the third Stokes parameter beam efficiency can exceed that of either polarization (first and second modified Stokes parameters), and when dualpolarized antenna patterns are equal, the fourth Stokes parameter beam efficiency is doubly degraded by antenna pattern cross polarization. These beam efficiencies, together with cross polarization and other Stokes parameter mixing ratios, are composed of elements from the integrated main-beam matrix. We also show that these results are applicable to both correlationtype and polarization-adding polarimeters and can be extended 


$$
\begin{aligned}
{\left[\begin{array}{llll}
m_{I I} & m_{I Q} & m_{I U} & m_{I V} \\
m_{Q I} & m_{Q Q} & m_{Q U} & m_{Q V} \\
m_{U I} & m_{U Q} & m_{U U} & m_{U V} \\
m_{V I} & m_{V Q} & m_{V U} & m_{V V}
\end{array}\right] } & =\left[\begin{array}{cccc}
1 & 1 & 0 & 0 \\
1 & -1 & 0 & 0 \\
0 & 0 & 1 & 0 \\
0 & 0 & 0 & 1
\end{array}\right]\left[\begin{array}{llll}
m_{v v} & m_{v h} & m_{v U} & m_{v V} \\
m_{h v} & m_{h h} & m_{h U} & m_{h V} \\
m_{U v} & m_{U h} & m_{U U} & m_{U V} \\
m_{V v} & m_{V h} & m_{V U} & m_{V V}
\end{array}\right]\left[\begin{array}{cccc}
\frac{1}{2} & \frac{1}{2} & 0 & 0 \\
\frac{1}{2} & -\frac{1}{2} & 0 & 0 \\
0 & 0 & 1 & 0 \\
0 & 0 & 0 & 1
\end{array}\right] \\
= & {\left[\begin{array}{ccccc}
\frac{1}{2}\left(m_{v v}+m_{v h}+m_{h v}+m_{h h}\right) & \frac{1}{2}\left(m_{v v}-m_{v h}+m_{h v}-m_{h h}\right) & m_{v U}+m_{h U} & m_{v V}+m_{h V} \\
\frac{1}{2}\left(m_{v v}+m_{v h}-m_{h v}-m_{h h}\right) & \frac{1}{2}\left(m_{v v}-m_{v h}-m_{h v}+m_{h h}\right) & m_{v U}-m_{h U} & m_{v V}-m_{h V} \\
\frac{1}{2}\left(m_{U v}+m_{U h}\right) & \frac{1}{2}\left(m_{U v}-m_{U h}\right) & m_{33} & m_{U V} & m_{43}
\end{array}\right] }
\end{aligned}
$$

TABLE I

SAMPLING OF NOTATIONS FOR STOKES PARAMETERS

\begin{tabular}{|c|c|c|c|c|c|c|c|}
\hline Specific & Intensity & $\begin{array}{c}1974 \\
{[29]}\end{array}$ & $\begin{array}{l}1991 \\
{[38]}\end{array}$ & $\begin{array}{c}1995 \\
{[2]}\end{array}$ & $\begin{array}{c}1998 \\
{[6]}\end{array}$ & $\begin{array}{c}2001 \\
{[3]}\end{array}$ & $\begin{array}{c}2003 \\
{[4]}\end{array}$ \\
\hline- & $I_{v}$ & $T_{v}$ & $T_{B v}$ & $T_{v}$ & $T_{V}$ & $T_{v}$ & $T_{v}$ \\
\hline - & $I_{h}$ & $T_{h}$ & $T_{B h}$ & $T_{h}$ & $T_{H}$ & $T_{h}$ & $T_{h}$ \\
\hline$s_{0}$ & $I$ & - & 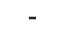 & $I$ & - & - & . \\
\hline$s_{1}$ & $Q$ & - & - & $Q$ & - & - & - \\
\hline$s_{2}$ & $U$ & - & $U_{B}$ & $U$ & $T_{3}$ & $T_{U}$ & $T_{3}$ \\
\hline$s_{3}$ & $V$ & - & $V_{B}$ & $V$ & $T_{4}$ & $T_{V}$ & $T_{4}$ \\
\hline
\end{tabular}
IN BRIGHTNESS TEMPERATURES

to any feed polarization through the use of a basis-transforming Mueller matrix.

The decomposition of the antenna pattern Mueller matrix into an integrated main-beam matrix and an additional sidelobe region leads to a potential polarimetric APC technique. Following the scalar technique presented in [24], the Stokes antenna temperatures can be corrected for sidelobe and cross polarization contamination to find a vector of effective main-beam Stokes brightness temperatures $\bar{T}_{\mathrm{MB}}$, which is defined as

$$
\bar{T}_{\mathrm{MB}}=\overline{\bar{\eta}}_{M}^{-1}\left[\bar{T}_{\mathrm{A}}-\underset{4 \pi-\text { main lobe }}{\int} \overline{\bar{M}}(\widehat{k}) \overline{\bar{R}}(\psi) \bar{T}_{\mathrm{B}}(\widehat{k}) d \Omega\right] .
$$

This correction takes care of all the cross polarizations and Stokes mixing in the main beam, as well as any Stokes mixing and polarization rotation present in the sidelobe region. We expect this correction approach, which is built upon the formulation for the Stokes antenna temperatures presented here, will be the fundamental basis of future polarimetric radiometer antenna calibration.

\section{APPENDIX}

A number of authors have introduced different notations for Stokes parameters of brightness temperatures. A sampling is summarized in Table I. The first column contains the conventional notation for the Stokes parameters in the specific intensity standard in the literature. The remaining columns give notations for the brightness temperatures that appear in chronological order from left to right and are denoted by first author and reference number. Note that we also included some early works that deal only with the vertical and horizontal po- larizations for completeness. The major differences between the notations occurred from the authors' concerted effort to avoid confusion between the vertical polarized or second modified Stokes brightness temperature and the fourth Stokes brightness temperature. In addition, there seems to be a mixed attempt to follow the convention in [24] to use uppercase subscripts for radiometric temperatures.

Sometimes, it is more convenient to operate in a (true) Stokes vector form, as opposed to a modified Stokes vector form. The Stokes vector is related to the modified Stokes vector in brightness temperature by [22]

$$
\left[\begin{array}{c}
T_{\mathrm{B}, I} \\
T_{\mathrm{B}, Q} \\
T_{\mathrm{B}, U} \\
T_{\mathrm{B}, V}
\end{array}\right]=\left[\begin{array}{c}
T_{\mathrm{B}, v}+T_{\mathrm{B}, h} \\
T_{\mathrm{B}, v}-T_{\mathrm{B}, h} \\
T_{\mathrm{B}, U} \\
T_{\mathrm{B}, V}
\end{array}\right]
$$

This relationship can be expressed as a transformation from modified to true Stokes vectors

$$
\left[\begin{array}{c}
T_{\mathrm{B}, I} \\
T_{\mathrm{B}, Q} \\
T_{\mathrm{B}, U} \\
T_{\mathrm{B}, V}
\end{array}\right]=\left[\begin{array}{cccc}
1 & 1 & 0 & 0 \\
1 & -1 & 0 & 0 \\
0 & 0 & 1 & 0 \\
0 & 0 & 0 & 1
\end{array}\right]\left[\begin{array}{c}
T_{\mathrm{B}, v} \\
T_{\mathrm{B}, h} \\
T_{\mathrm{B}, U} \\
T_{\mathrm{B}, V}
\end{array}\right]
$$

In the reverse direction, this is

$$
\left[\begin{array}{l}
T_{\mathrm{B}, v} \\
T_{\mathrm{B}, h} \\
T_{\mathrm{B}, U} \\
T_{\mathrm{B}, V}
\end{array}\right]=\left[\begin{array}{cccc}
\frac{1}{2} & \frac{1}{2} & 0 & 0 \\
\frac{1}{2} & -\frac{1}{2} & 0 & 0 \\
0 & 0 & 1 & 0 \\
0 & 0 & 0 & 1
\end{array}\right]\left[\begin{array}{c}
T_{\mathrm{B}, I} \\
T_{\mathrm{B}, Q} \\
T_{\mathrm{B}, U} \\
T_{\mathrm{B}, V}
\end{array}\right] .
$$

The aforementioned equation can be applied to Mueller matrices as well. A Mueller matrix (for a modified Stokes vector) is transformed to its "nonmodified" format expressed in (66), shown at the top of the page.

\section{REFERENCES}

[1] M. S. Dzura, V. S. Etkin, A. S. Khrupin, M. S. Pospelov, and M. D. Raev, "Radiometers-polarimeters: Principles of design and applications for sea surface microwave emission polarimetry," in Proc. IGARSS, Houston, TX, 1992, pp. 1432-1434.

[2] S. Yueh, W. Wilson, F. Li, S. Nghiem, and W. Ricketts, "Polarimetric measurements of sea surface brightness temperatures using an aircraft K-band radiometer," IEEE Trans. Geosci. Remote Sens., vol. 33, no. 1, pp. 85-92, Jan. 1995. 
[3] J. R. Piepmeier and A. J. Gasiewski, "Digital correlation microwave polarimetry: Analysis and demonstration," IEEE Trans. Geosci. Remote Sens., vol. 39, no. 11, pp. 2392-2410, Nov. 2001.

[4] J. Lahtinen, J. Pihlflyckt, I. Mononen, S. Tauriainen, M. Kemppinen, and M. Hallikainen, "Fully polarimetric microwave radiometer for remote sensing," IEEE Trans. Geosci. Remote Sens., vol. 41, no. 8, pp. 18691878, Aug. 2003.

[5] P. Gaiser, K. St. Germain, E. Twarog, G. Poe, W. Purdy, D. Richardson, W. Grossman, W. Jones, D. Spencer, G. Golba, J. Cleveland, L. Choy, R. Bevilacqua, and P. Chang, "The WindSat spaceborne polarimetric microwave radiometer: Sensor description and early orbit performance," IEEE Trans. Geosci. Remote Sens., vol. 42, no. 11, pp. 2347-2361, Nov. 2004.

[6] C. Ruf, "Constraints on the polarization purity of a Stokes microwave radiometer," Radio Sci., vol. 33, no. 6, pp. 1617-1639, Nov./Dec. 1998.

[7] Y. Kerr, P. Waldteufel, J. Wigneron, J. Martinuzzi, J. Font, and M. Berger, "Soil moisture retrieval from space: The Soil Moisture and Ocean Salinity (SMOS) mission," IEEE Trans. Geosci. Remote Sens., vol. 39, no. 8, pp. 1729-1735, Aug. 2001.

[8] D. L. Vine, G. Lagerloef, F. Colomb, and Y. Chao, "Aquarius: A passive/active microwave sensor to monitor sea surface salinity globally from space," in Proc. SPIE Microw. Remote Sens. Environ. IV, 2004, vol. 5654, pp. 122-128.

[9] National Research Council, Earth Science and Applications From Space: National Imperatives for the Next Decade and Beyond, 2007, Washington DC: Nat. Acad. Press.

[10] D. Entekhabi, E. G. Enjoku, P. Houser, M. Spencer, T. Doiron, Y. Kim, J. Smith, R. Girard, S. Belair, W. Crow, T. J. Jackson, Y. H. Kerr, J. S. Kimball, R. Koster, K. C. McDonald, P. E. O'Neill, T. Pultz, S. W. Running, J. Shi, E. Wood, and J. van Zyl, "The Hydrosphere State (Hydros) satellite mission: An Earth system pathfinder for global mapping of soil moisture and land freeze/thaw," IEEE Trans. Geosci. Remote Sens., vol. 42, no. 10, pp. 2184-2195, Oct. 2004.

[11] S. H. Yueh, "Estimates of Faraday rotation with passive microwave polarimetry for microwave remote sensing of Earth surfaces," IEEE Trans. Geosci. Remote Sens., vol. 38, no. 5, pp. 2434-2438, Sep. 2000.

[12] D. Hudson, J. Piepmeier, and D. Long, "Polarization rotation correction in radiometry: An extended error analysis," in Proc. IGARSS, Denver, CO, Aug. 2006, pp. 2305-2308.

[13] M. Martin-Neira, S. Ribo, and A. J. Martin-Polegre, "Polarimetric mode of MIRAS," IEEE Trans. Geosci. Remote Sens., vol. 40, no. 8, pp. 17551768, Aug. 2002.

[14] J. Piepmeier and N. Simon, "A polarimetric extension of the van Cittert-Zernike theorem for use with microwave interferometers," IEEE Geosci. Remote Sens. Lett., vol. 1, no. 4, pp. 300-303, Oct. 2004.

[15] I. Corbella, A. J. Gasiewski, M. Klein, and J. R. Piepmeier, "Compensation of elevation angle variations in polarimetric brightness temperature measurements from airborne microwave radiometers," IEEE Trans. Geosci. Remote Sens., vol. 39, no. 1, pp. 193-195, Jan. 2001.

[16] T. Meissner and F. Wentz, "Polarization rotation and the third Stokes parameter: The effects of spacecraft attitude and Faraday rotation," IEEE Trans. Geosci. Remote Sens., vol. 44, no. 3, pp. 506-515, Mar. 2006.

[17] A. J. Gasiewski and D. B. Kunkee, "Calibration and applications of polarization-correlating radiometers," IEEE Trans. Microw. Theory Tech., vol. 41, no. 5, pp. 767-773, May 1993.

[18] M. Spencer, E. Njoku, D. Entekhabi, T. Doiron, J. Piepmeier, and R. Girard, "The HYDROS radiometer/radar instrument," in Proc. IGARSS, Anchorage, AK, 2004, pp. 691-694.

[19] S. Sobjaerg and N. Skou, "Polarimetric signatures from a crop covered land surface measured by an L-band polarimetric radiometer," in Proc. IGARSS, Toulouse, France, Jul. 2003, pp. 2626-2628.

[20] B. Hornbuckle, A. England, R. De Roo, M. Fischman, and D. Boprie, "Vegetation canopy anisotropy at $1.4 \mathrm{GHz}$, , IEEE Trans. Geosci. Remote Sens., vol. 41, no. 10, pp. 2211-2223, Oct. 2003.

[21] P. Narvekar, T. Jackson, R. Bindlish, L. Li, G. Heygster, and P. Gaiser, "Observations of land surface passive polarimetry with the WindSat instrument," IEEE Trans. Geosci. Remote Sens., vol. 45, no. 7, pp. 20192028, Jul. 2007.

[22] J. D. Kraus, Radio Astronomy, 2nd ed. Powell, OH: Cygnus-Quasar Books, 1986.

[23] J. Tinbergen, Astronomical Polarimetry. New York: Cambridge Univ. Press, 1996.

[24] F. T. Ulaby, R. K. Moore, and A. K. Fung, Microwave Remote Sensing: Active and Passive. Reading, MA: Addison-Wesley, 1986.

[25] Y.-Q. Jin, Electromagnetic Scattering Modelling for Quantitative Remote Sensing. River Edge, NJ: World Scientific, 1993.
[26] H. Ko, "On the reception of quasi-monochromatic, partially polarized radio waves," Proc. IRE, vol. 50, no. 9, pp. 1950-1957, Sep. 1962.

[27] M. Born and E. Wolf, Principles of Optics, 7th ed. New York: Cambridge Univ. Press, 1999

[28] H. Ko, "The use of the statistical matrix and the Stokes vector in formulating the effective aperture of antennas," IEEE Trans. Antennas Propag., vol. AP-9, no. 6, pp. 581-582, Nov. 1961.

[29] J. Claassen and A. Fung, "The recovery of polarized apparent temperature distributions of flat scenes from antenna temperature measurements," IEEE Trans. Antennas Propag., vol. AP-22, no. 3, pp. 433-442, May 1974.

[30] E. Njoku, "Antenna pattern correction procedures for the Scanning Multichannel Microwave Radiometer (SMMR)," Bound.-Layer Meteorol., vol. 18, no. 1, pp. 79-98, Feb. 1980.

[31] S. Chandrasekhar, Radiative Transfer. New York: Dover, 1960.

[32] L. Tsang, J. A. Kong, and R. T. Shin, Theory of Microwave Remote Sensing. New York: Wiley, 1985.

[33] J. P. Hamaker, J. D. Bregman, and R. J. Sault, "Understanding radio polarimetry_Part I: Mathematical foundations," Astron. Astrophys., Suppl. Ser., vol. 117, no. 1, pp. 137-147, 1996.

[34] R. Schmeider, "Stokes-algebra formalism," J. Opt. Soc. Amer., vol. 59, no. 3, pp. 297-302, Mar. 1969.

[35] A. C. Ludwig, "The definition of cross polarization," IEEE Trans. Antennas Propag., vol. AP-21, no. 1, pp. 116-119, Jan. 1973.

[36] G. Sinclair, "The transmission and reception of elliptically polarized waves," Proc. IRE, vol. 38, no. 2, pp. 148-151, Feb. 1950.

[37] C. Heiles, P. Perillat, M. Nolan, D. Lorimer, R. Bhat, T. Ghosh, M. Lewis, K. O'Neil, C. Salter, and S. Stanimirovic, "Mueller matrix parameters for radio telescopes and their observational determination," Publ. Astron. Soc. Pac., vol. 113, no. 788, pp. 1274-1288, Oct. 2001.

[38] L. Tsang, "Polarimetric passive microwave remote sensing of random discrete scatterers and rough surfaces," J. Electromagn. Waves Appl., vol. 5, no. 1, pp. 41-57, 1991.

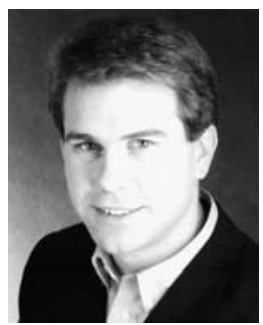

Jeffrey R. Piepmeier (S'93-M'99) received the B.S. degree in engineering from LeTourneau University, Longview, TX, in 1993 and the M.S. and Ph.D. degrees in electrical engineering from the Georgia Institute of Technology, Atlanta, in 1994 and 1999, respectively.

From 1993 to 1994, he was a Schakleford Fellow with the Georgia Tech Research Institute. In 1999, he joined the Microwave Instrument Technology Branch, Goddard Space Flight Center (GSFC), National Aeronautics and Space Administration (NASA), Greenbelt, MD, as a Principal Investigator on several technology development projects in microwave radiometry. During 2004-2005, he led the NASA Hydrosphere State (Hydros) Radiometer Team. He is currently a Calibration Lead for the NASA GSFC Aquarius radiometer.

Dr. Piepmeier is a member of the Union Radio Scientifique Internationale Commission $\mathrm{F}$ and the American Geophysical Union. He is a past Chair of the Geoscience and Remote Sensing Society Instrumentation and Future Technologies Technical Subcommittee, the past Conference Chair of the 2000 Microwave Radiometer Calibration Workshop, and a 2002 NASA Earth Science New Investigator. He is currently the Vice-Chair of the National Academies' Committee on Radio Frequencies. He received the Third Place in the International Geoscience and Remote Sensing Symposium Student Prize Paper Competition in 1998, the Excellence in Federal Career Gold Award (Rookie of the Year) in 2000, and the NASA GSFC Exceptional Achievement Award and Technology Advancement Award in 2005 and 2007, respectively. 


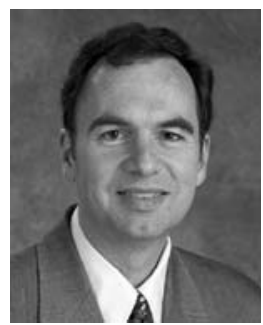

David G. Long (S'84-M'89-SM'98) received the Ph.D. degree in electrical engineering from the University of Southern California, Los Angeles, in 1989.

From 1983 to 1990, he was with the Jet Propulsion Laboratory (JPL), National Aeronautics and Space Administration (NASA), where he developed advanced radar remote sensing systems. While at JPL, he was the Project Engineer on the NASA Scatterometer (NSCAT) Project, which flew from 1996 to 1997. He also managed the Scanned SCAT Project, the precursor to SeaWinds, which was launched in 1999 and 2002. He is currently a Professor in the Department of Electrical and Computer Engineering, Brigham Young University (BYU), Provo, UT, where he teaches upper division and graduate courses in communications, microwave remote sensing, radar, and signal processing and where he is also the Director of the Center for Remote Sensing. He is also the Principal Investigator for several NASA-sponsored research projects in remote sensing. His research interests include microwave remote sensing, radar theory, space-based sensing, estimation theory, signal processing, and mesoscale atmospheric dynamics. $\mathrm{He}$ is the author or coauthor of nearly 300 publications.

Dr. Long is an Associate Editor for the IEEE GeOscIENCE AND REMOTE SENSING LETTERS. He received the NASA Certificate of Recognition several times.

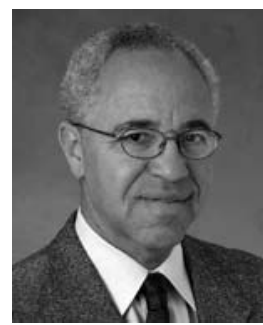

Eni G. Njoku (M'75-SM'83-F'95) received the B.A. degree in natural and electrical sciences from Cambridge University, Cambridge, U.K., in 1972 and the M.S. and Ph.D. degrees in electrical engineering from the Massachusetts Institute of Technology, Cambridge, in 1974 and 1976, respectively.

From 1976 to 1977, he was a National Research Council Postdoctoral Research Associate. In 1977, he joined the Jet Propulsion Laboratory (JPL), California Institute of Technology, Pasadena, where he is currently a Senior Research Scientist and the Supervisor of the Water and Carbon Cycles Group. He is a Member of the Earth Observing System Aqua Advanced Microwave Scanning Radiometer Science Team and was the JPL Project Scientist for the Hydrosphere State (Hydros) Mission. From 1986 to 1990, he served as a Discipline Scientist for ocean and Earth science data systems at the National Aeronautics and Space Administration Headquarters, Washington, DC, and from 1993 to 1994, he was the Manager of the Geology and Planetology Section at JPL. During the 2001-2002 academic year, he was on leave as a Visiting Professor in the Department of Civil and Environmental Engineering, Massachusetts Institute of Technology. His primary interests are the applications of microwave remote sensing to land surface hydrology and climate. His research involves microwave modeling of land and ocean surfaces, retrieval algorithm development, satellite and airborne sensor data analysis for hydrologic applications, and development of new spaceborne observing concepts.

Dr. Njoku is a member of the American Meteorological Society, American Geophysical Union, American Association for the Advancement of Science, Union Radio Scientifique Internationale Commission F, and Sigma Xi. He has served as an Associate Editor for the IEEE TRANSACTIONS ON GEOSCIENCE AND Remote Sensing (1985-1988), the Technical Program Chairman for the 1994 International Geoscience and Remote Sensing Symposium, and a member of the Geoscience and Remote Sensing Society Administrative Committee (1997-2000). He is the recipient of the 1980, 1982, and 1985 NASA Group Achievement Awards and the 1985 NASA Exceptional Service Medal. 